\title{
The differentiation of rhythmic structure
}

\author{
STEPHEN HANDEL \\ University of Tennessee, Knoxville, Tennessee
}

\begin{abstract}
Listeners judged whether two five-tone nonmetric rhythms were the same or different. Each rhythm was presented one, two, or four times to study the process of perceptual differentiation. The results indicated that the listeners perceived these rhythms in terms of the grouping of the tones, and not in terms of the timing between the groups. Two rhythms that had the same perceptual grouping were judged as being identical, even if the timing between the groups was different. The perception of the groupings of tones developed gradually. If each rhythm was presented only once, then the listeners had only a global percept, focused on groups (runs) of three elements, and often judged two different rhythms as being identical. If the rhythms were presented two or four times, then the grouping of the tones became more differentiated and the listeners were less likely to judge different patterns as being identical. Thus, perception of auditory rhythmic structure appears to follow the same developmental process as the perception of visual spatial structure.
\end{abstract}

The purpose of the present paper is to describe the stages by which listeners come to perceive rhythmic structure. By their very nature, rhythms unfold in time and each repetition provides a single glimpse into the structure. Although each repetition is physically complete, perceptually, the rhythm seems to coalesce over time. The fundamental issue is to understand the progression toward the final stable percept.

This progression was originally studied in vision by Krueger and Sander (described in Sander, 1930), who termed this process of organization aktualgenese, and was continued theoretically by Heinz Werner, who translated this term as microgenesis (Flavell \& Draguns, 1957; Smith, 1957). For these theorists, the initial stages consist of diffuse, undifferentiated, and equivocal figureground percepts. The inner contents of the figure remain vague and amorphous. At this point, there are numerous possibilities for further differentiation. A tentative, labile configuration then emerges and, finally, elaboration and modification of the skeletal gestalt yields the ultimate percept. The stage preceding the formation of the final stable percept (Vorgestalt, or preconfiguration stage) is more undifferentiated internally, more regular, and simpler in form and content than the final percept is.

The experimental question is to abstract both the global, more regular rhythmic structures that are perceived initially, and the subsequent differentiation of the specific timing structure. The assumption has always been that the initial percept contains the germ of the correct structure. If this is the case, at the beginning, listeners ought to perceive the rhythm as one of many alternatives, and suc-

I thank Bonnie Hiller and Nathan Wright for their help in running the experiment and Carol Krumhansl, Schuyler Huck, and the reviewers for their helpful comments on the manuscript. Correspondence should be addressed to S. Handel, Department of Psychology, University of Tennessee, Knoxville, TN 37996-0900. cessive repetitions ought to prune away the incorrect ones. It is this partitioning that provides insights into rhythmic differentiation.

In the present experiment, we used a same-different task with nonmetric rhythms. In this task, one rhythm was presented using lower pitch tones and a second rhythm was presented using higher pitch tones; the subject's task was to judge whether the two rhythms were the same or different. By varying the presentation conditions, it is possible to investigate the differentiation of the percept. At one extreme, the first pattern (A) would be presented once and then the second pattern (either the identical Pattern A or a different pattern, $B$ ) would be presented once (we will use the notation $A X$ to indicate that either $A A$ or $A B$ could be presented). At the other extreme, the first pattern would be presented four times and then the second pattern would be presented four times (e.g., AAAAXXXX); the first and second patterns could be alternated four times (e.g., AXAXAXAX) or the first and second patterns could be alternated in blocks of two (e.g., AAXXAAXX). For intermediate cases, the first pattern would be presented two times and the second pattern would be presented twice (e.g., AAXX) or the first and second pattern could be alternated twice (e.g., AXAX).

We selected these conditions for two reasons. First, we expected the confusions (i.e., judging two identical patterns as being different or two different patterns as being identical) to change across presentation conditions. For the condition in which each rhythm is presented once only, the rhythmic structure ought to be undifferentiated and many confusions should occur. For the conditions in which each rhythm is presented four times, the rhythmic structure ought to be more differentiated and only a small number of confusions should occur. By considering the changes in the confusions, the process of differentiation can be analyzed. Second, we expected that the way the comparison patterns are presented in terms of the num- 
ber of alternations and the number of repetitions between alternations would affect performance. For this reason, even though each rhythm is presented four times in the conditions AXAXAXAX, AAXXAAXX, and AAAAXXXX, there may be large differences in discrimination as a function of rhythm difficulty or tempo.

To study the differentiation in its clearest form, nonmetric patterns based on unfilled intervals, as opposed to typical Western metric patterns, were chosen. Meter is the sense of a regular periodic sequence of subjectively stronger and weaker beats that characterize music. The meter occurs at several hierarchical levels at once, such that the beats at higher levels occur at integer multiples of the beats at the lower levels. For example, suppose we have a rhythm based on a repeating unit of 16 elements. At the lower level, the beats would occur at every element or every other element; at a higher level, beats would occur at every fourth element (e.g., 1, 5, 9, and 13); at a still higher level, beats would occur at every eighth element (e.g., 1 and 9); and at the highest level, the beats would occur only at the beginning of each repetition. The strength of any beat/element is determined by the number of levels at which the beat appears. Thus, the strong beats occur at Elements 1, 5, 9, and 13, stronger beats occur at Elements 1 and 9, and the strongest beat occurs at Element 1. Because the beats at different levels occur at integer multiples, it is natural to expect that the intervals between elements (i.e., from the onset of one tone to the onset of the next tone) would also occur at integer multiples, namely, 1:2 or 1:3. In fact, Fraisse (1982) found that a shorter interval and a longer interval account for $85 \%$ of the durations in a piece; this is true across a wide variety of music. The longer interval is typically 2 or 3 times longer than the shorter. Converging evidence comes from the findings of Povel (1981) and Essens and Povel (1985) in which subjects could most accurately reproduce $1: 2$ intervals.

The meter forms a time-based lattice that serves to create the rhythmic organization. Notes that fall at the points of the strong beats become accented and notes that fall at the points of the weak beats become unaccented. Rhythmic organization, therefore, is fitting the meter grid to the note sequence. One would start with the grid of strong and weak beats and "slide" the grid along the passage so that the strong beats fall on different notes as the grid moves. The problem then is to select the best meter for a note sequence. Several theorists (Cooper \& Meyer, 1960; Lerdahl \& Jackendoff, 1983; Longuet-Higgens \& Lee, 1982, 1984; Povel \& Essens, 1985) have suggested preference rules for fitting the meter grid to a passage. Here, we will make use of the rules suggested by Povel and Essens for sequences of identical elements separated by different lengths of silent intervals, such as $x . x . . x . x . . x \ldots$ the xs represent tone elements, the dots represent isochronous silent time intervals). Two of their rules are most critical: (1) a strong meter beat should not occur on a silence or rest, and (2) a strong beat should occur on the first and last tone of a run of adjacent identical tones. We will operationally define metric patterns as those that satisfy these two rules. Thus, the rhythm $x \cdot x \cdot x \ldots x \ldots x \ldots$ would be metric because tones fall on the stronger beats at Positions 1, 5, 9, and 13, but the rhythm $x . x \ldots x . x . x \ldots$. would be nonmetric because tones do not fall at Positions 5,9 , or 13 . From this perspective, rhythms are not simply metric or nonmetric; instead, each rhythm is metric to some degree, depending on the strength of the metric interpretation it evokes. Here, we will define the metric strength by the occurrence of tones at Positions 1, 5, 9, and 13. Povel and Essens, among others, argue that listeners attempt to find a meter to fit a rhythmic pattern and that highly metric patterns more easily induce an internal clock that codes the rhythm temporally. Thus, these rhythms would be easier to represent and reproduce.

We chose to investigate the perception of nonmetric rhythms to understand the differentiation of the timing structure without the use of an intervening, preexisting schema (Palmer \& Krumhansl, 1990). The use of metric rhythms tends to restrict the possible timings between elements. The resulting hierarchical framework might lead listeners to hear the rhythms only in specific ways, and thereby limit our understanding of perceptual differentiation. According to the above views, nonmetric patterns do not induce an internal clock and, therefore, the rhythm cannot be coded in terms of a temporal grid in which every element can be located and timed. Instead, these rhythms are organized into bounded groups of elements that follow one another, although the timings between the onsets of the successive groups are not encoded or compared. Bamberger (1978) and Povel and Essens (1985) term this figural grouping because the groups are figures perceived against an ordinal, ongoing time. For example, the nonmetric pattern illustrated above, $x . x \ldots x . x . x \ldots$, would be coded as 2 , silence, 1 , silence, 2 , silence, and the lengths of the silent intervals would be coded only roughly, if at all. We will write this grouping as 2-1-2. Thus, we might expect that the similar nonmetric pattern $\mathrm{x} . \mathrm{x} \ldots \mathrm{x} \ldots \mathrm{x} . \mathrm{x}$.... would be coded identically as 2 , silence, 1 , silence, 2 , silence and, therefore, would be easily confused with the former rhythm.

The rhythms are shown in Table 1 . All are 16 units long; the locations of the strong and weak accents are indicated by asterisks. The rhythms are nonmetric in two ways. First, no rhythm has tones that occur on all the stronger beat elements: $1,5,9$, and 13 . One rhythm (4) has tones at three of the four stronger beat elements: 1 , 5 , and 9 . Seven rhythms $(2,3,6,10,11,12$, and 13$)$ have tones only at the strongest beat at the beginning of the rhythm. Second, the onset-to-onset intervals occur in 2:3:4 ratios so that the ratio between intervals is a simple integer in only one case.

Another factor that probably affects rhythmic differentiation is the tempo or rate of presentation. Research by Garner (1974), Handel and Lawson (1983), Monahan and Hirsh (1990), and Ten Hoopen (1992), using diverse stimuli and procedures, have found that rhythmic organization changes at different presentation rates. For this rea- 
Table 1

Average Ratings (AR) and Percent Correct (PC) for all Pairs of Rhythms at the Slow (1.9 Elements/Sec) Presentation Rate

\begin{tabular}{|c|c|c|c|c|c|c|c|c|}
\hline \multirow{3}{*}{$\begin{array}{c}\text { Pair } \\
\text { Number } \neq\end{array}$} & \multirow[b]{3}{*}{ Rhythm $\dagger$} & \multirow{3}{*}{$\begin{array}{c}\text { Rhythms } \\
\text { Timing } \\
\end{array}$} & \multicolumn{6}{|c|}{ Alternation Conditions } \\
\hline & & & \multicolumn{2}{|c|}{$\begin{array}{c}\text { AX } \\
\text { AXAX }\end{array}$} & \multicolumn{2}{|c|}{$\begin{array}{c}\text { AAXX } \\
\text { AAXXAAXX } \\
\text { AAAAXXXX } \\
\end{array}$} & \multicolumn{2}{|c|}{ AXAXAXAX } \\
\hline & & & $\overline{\mathrm{AR}}$ & PC & AR & PC & AR & PC \\
\hline & & $\begin{array}{llllll}* & & & & \\
* & & & * & \\
* & * & * & *\end{array}$ & & & & & & \\
\hline \multicolumn{9}{|c|}{ Identical Rhythms } \\
\hline & 1 & $x \cdot x \cdot x \ldots x \ldots x \ldots$ & 1.4 & 90 & 1.4 & 95 & 1.3 & 89 \\
\hline & 2 & $\mathbf{x} \cdot \mathbf{x} \ldots \mathbf{x} \cdot \mathbf{x} \ldots \mathbf{x} \ldots$ & 1.5 & 87 & 1.3 & 93 & 1.2 & 100 \\
\hline & 3 & $x \cdot x \ldots x \ldots x \cdot x \ldots$ & 1.7 & 82 & 1.5 & 87 & 1.5 & 85 \\
\hline & 4 & $\mathbf{x} \cdot \mathbf{x} \cdot \mathbf{x} \ldots \mathbf{x} \ldots \mathbf{x} \ldots$ & 1.2 & 98 & 1.2 & 97 & 1.1 & 100 \\
\hline & 5 & $x \cdot x \ldots x \cdot x \ldots x \ldots$ & 1.4 & 90 & 1.3 & 91 & 1.1 & 96 \\
\hline & 6 & $\mathbf{x} \cdot \mathbf{x} \ldots \mathbf{x} \ldots \mathbf{x} \cdot \mathbf{x} \ldots$ & 1.5 & 85 & 1.4 & 92 & 1.2 & 96 \\
\hline & 7 & $\mathbf{x} \cdot \mathbf{x} \ldots \mathbf{x} \ldots \mathbf{x} \ldots$ & 1.4 & 94 & 1.4 & 91 & 1.4 & 89 \\
\hline & 8 & $x \ldots x \cdot x \ldots x \ldots x \ldots$ & 1.4 & 89 & 1.4 & 91 & 1.3 & 93 \\
\hline & 9 & $x \ldots x \ldots x . x \ldots x \ldots$ & 1.5 & 89 & 1.4 & 92 & 1.3 & 95 \\
\hline & 10 & $\mathbf{x} \ldots \mathbf{x} \ldots \mathbf{x} \ldots \mathbf{x} \ldots$ & 1.4 & 91 & 1.3 & 93 & 1.2 & 100 \\
\hline & 11 & $\mathbf{x} \ldots \mathbf{x} \cdot \mathbf{x} \cdot \mathbf{x} \ldots \mathbf{x} \ldots$ & 1.3 & 91 & 1.4 & 88 & 1.1 & 96 \\
\hline & 12 & $x \ldots x \cdot x \ldots x \cdot x \ldots$ & 1.2 & 96 & 1.5 & 87 & 1.3 & 93 \\
\hline & 13 & $\mathbf{x} \ldots \mathbf{x} \ldots \mathbf{x} \cdot \mathbf{x} \cdot \mathbf{x} \ldots$ & 1.4 & 91 & 1.2 & 96 & 1.2 & \\
\hline
\end{tabular}

\section{Different Rhythms}

\begin{tabular}{|c|c|c|c|c|c|c|c|c|}
\hline 14 & 1 & $\mathbf{x} \cdot \mathbf{x}, \mathbf{x} \ldots \mathbf{x} \ldots \mathbf{x} \ldots$ & 1.5 & 9 & 2.3 & 41 & 1.9 & 30 \\
\hline & 4 & $x \cdot x \cdot x \ldots x \ldots x \ldots$ & & & & & & \\
\hline 15 & 2 & $\mathbf{x} \cdot \mathbf{x} \ldots \mathbf{x} \cdot \mathbf{x} \ldots \mathbf{x} \ldots$ & 1.6 & 15 & 2.0 & 32 & 1.9 & 30 \\
\hline & 5 & $\mathbf{x} \cdot \mathbf{x} \ldots \mathbf{x} \cdot \mathbf{x} \ldots \mathbf{x} \ldots$ & & & & & & \\
\hline 16 & 6 & $x \cdot x \ldots x \ldots x \cdot x \ldots$ & 1.3 & 6 & 1.9 & 24 & 1.8 & 22 \\
\hline & 3 & $\mathbf{x} \cdot \mathbf{x} \ldots \mathbf{x} \ldots \mathbf{x} \cdot \mathbf{x} \ldots$ & & & & & & \\
\hline$M$ & & & 1.5 & 10 & 2.1 & 32 & 1.9 & 27 \\
\hline 17 & 6 & $\mathbf{x}, \mathbf{x} \ldots \mathbf{x} \ldots \mathbf{x}, \mathbf{x} \ldots$ & 2.5 & 50 & 3.0 & 70 & 3.6 & 93 \\
\hline & 7 & $x \cdot x \ldots x \ldots x \ldots x \ldots$ & & & & & & \\
\hline 18 & 8 & $\mathbf{x} \ldots \mathbf{x} \cdot \mathbf{x} \ldots \mathbf{x} \ldots \mathbf{x} \ldots$ & 2.7 & 60 & 2.7 & 60 & 3.6 & 93 \\
\hline & 12 & $\mathbf{x} \ldots \mathbf{x} \cdot \mathbf{x} \ldots \mathbf{x} \cdot \mathbf{x} \ldots$ & & & & & & \\
\hline 19 & 8 & $\mathbf{x} \ldots \mathbf{x} \cdot \mathbf{x} \ldots \mathbf{x} \ldots \mathbf{x} \ldots$ & 2.7 & 62 & 2.8 & 69 & 3.5 & 89 \\
\hline & 9 & $x \ldots x \ldots x, x \ldots x \ldots$ & & & & & & \\
\hline 20 & 7 & $\mathbf{x} \cdot \mathbf{x} \ldots \mathbf{x} \ldots \mathbf{x} \ldots \mathbf{x} \ldots$ & 3.0 & 67 & 2.8 & 63 & 3.2 & 74 \\
\hline & 2 & $\mathbf{x} \cdot \mathbf{x} \ldots \mathbf{x} \cdot \mathbf{x} \ldots \mathbf{x} \ldots$ & & & & & & \\
\hline$M$ & & & 2.7 & 60 & 2.8 & 66 & 3.5 & 87 \\
\hline 21 & 10 & $\mathbf{x} \ldots \mathbf{x} \ldots \mathbf{x} \ldots \mathbf{x} \cdot \mathbf{x} \ldots$ & 2.8 & 60 & 3.3 & 78 & 3.4 & 78 \\
\hline & 8 & $x \ldots x, x \ldots x \ldots x \ldots$ & & & & & & \\
\hline 22 & 13 & $x \ldots x \ldots x \cdot x \cdot x \ldots$ & 2.8 & 60 & 3.6 & 89 & 3.7 & 87 \\
\hline & 5 & $\mathbf{x} \cdot \mathbf{x} \ldots \mathbf{x} \cdot \mathbf{x} \ldots \mathbf{x} \ldots$ & & & & & & \\
\hline 23 & 13 & $\mathbf{x} \ldots \mathbf{x} \ldots \mathbf{x} \cdot \mathbf{x} \cdot \mathbf{x} \ldots$ & 2.9 & 54 & 3.3 & 79 & 3.3 & 81 \\
\hline & 6 & $x \cdot x \ldots x \ldots x \cdot x \ldots$ & & & & & & \\
\hline 24 & 12 & $x \ldots x \cdot x \ldots x \cdot x \ldots$ & 2.9 & 65 & 3.1 & 73 & 3.6 & 89 \\
\hline & 3 & $\mathbf{x} \cdot \mathbf{x} \ldots \mathbf{x} \ldots \mathbf{x} \cdot \mathbf{x} \ldots$ & & & & & & \\
\hline 25 & 9 & $x \ldots x \ldots x \cdot x \ldots x \ldots$ & 3.0 & 65 & 3.3 & 81 & 3.8 & 97 \\
\hline & 13 & $x \ldots x \ldots x \cdot x \cdot x \ldots$ & & & & & & \\
\hline 26 & 2 & $\mathbf{x} \cdot \mathbf{x} \ldots \mathbf{x} \cdot \mathbf{x} \ldots \mathbf{x} \ldots$ & 3.0 & 67 & 3.1 & 72 & 3.7 & 93 \\
\hline & 9 & $\mathbf{x} \ldots \mathbf{x} \ldots \mathbf{x}, \mathbf{x} \ldots \mathbf{x} \ldots$ & & & & & & \\
\hline$M$ & & & 2.9 & 62 & 3.3 & 79 & 3.6 & 88 \\
\hline 27 & 11 & $x \ldots x \cdot x \cdot x \ldots x \ldots$ & 3.0 & 73 & 3.5 & 86 & 3.8 & 97 \\
\hline & 1 & $\mathbf{x} \cdot \mathbf{x} \cdot \mathbf{x} \ldots \mathbf{x} \ldots \mathbf{x} \ldots$ & & & & & & \\
\hline 28 & 11 & $\mathbf{x} \ldots \mathbf{x} \cdot \mathbf{x} \cdot \mathbf{x} \ldots \mathbf{x} \ldots$ & 3.1 & 70 & 3.5 & 85 & 4.0 & 100 \\
\hline & 2 & $\mathbf{x} \cdot \mathbf{x} \ldots \mathbf{x} \cdot \mathbf{x} \ldots \mathbf{x} \ldots$ & & & & & & \\
\hline 29 & 4 & $x, x, x \ldots x \ldots x \ldots$ & 3.2 & 69 & 3.3 & 81 & 3.6 & \\
\hline & 8 & $\mathbf{x} \ldots \mathbf{x} \cdot \mathbf{x} \ldots \mathbf{x} \ldots \mathbf{x} \ldots$ & & & & & & \\
\hline
\end{tabular}


Table 1(Continued)

\begin{tabular}{|c|c|c|c|c|c|c|c|c|}
\hline \multirow{3}{*}{$\begin{array}{c}\text { Pair } \\
\text { Number } \ddagger\end{array}$} & \multirow[b]{3}{*}{ Rhythm $\dagger$} & \multirow{3}{*}{$\begin{array}{c}\text { Rhythms } \\
\text { Timing }\end{array}$} & \multicolumn{6}{|c|}{ Alternation Conditions } \\
\hline & & & \multicolumn{2}{|c|}{$\underset{\text { AXAX }}{\mathrm{AXX}}$} & \multicolumn{2}{|c|}{$\begin{array}{c}\text { AAXX } \\
\text { AAXXAAXX } \\
\text { AAAAXXXX }\end{array}$} & \multicolumn{2}{|c|}{ AXAXAXAX } \\
\hline & & & AR & PC & AR & PC & AR & PC \\
\hline & & $\begin{array}{llll}* & & & \\
* & & * & \\
* & * & * & *\end{array}$ & & & & & & \\
\hline 30 & $\begin{array}{r}9 \\
10\end{array}$ & $\begin{array}{l}x \ldots x \ldots x \cdot x \ldots x \ldots \\
x \ldots x \ldots x \ldots x \cdot x \ldots\end{array}$ & 3.2 & 73 & 3.5 & 87 & 3.8 & 93 \\
\hline 31 & $\begin{array}{l}9 \\
7\end{array}$ & $\begin{array}{l}x \ldots x \ldots x \cdot x \ldots x \ldots \\
x \cdot x \ldots x \ldots x \ldots x \ldots\end{array}$ & 3.2 & 75 & 3.3 & 82 & 3.8 & 97 \\
\hline 32 & $\begin{array}{r}10 \\
6\end{array}$ & $\begin{array}{l}x \ldots x \ldots x \ldots x \ldots \\
x \cdot x \ldots x \ldots x \cdot x \ldots\end{array}$ & 3.2 & 75 & 3.3 & 75 & 3.5 & 85 \\
\hline 33 & $\begin{array}{l}5 \\
9\end{array}$ & $\begin{array}{l}x \cdot x \ldots x \cdot x \ldots x \ldots \\
x \ldots x \ldots x \cdot x \ldots x \ldots\end{array}$ & 3.2 & 81 & 3.5 & 86 & 3.6 & 89 \\
\hline 34 & $\begin{array}{l}3 \\
4\end{array}$ & $\begin{array}{l}x \cdot x \ldots x \ldots x \cdot x \ldots \\
x \cdot x \cdot x \ldots x \ldots x \ldots\end{array}$ & 3.4 & 88 & 3.5 & 83 & 3.8 & 93 \\
\hline 35 & $\begin{array}{l}12 \\
11\end{array}$ & $\begin{array}{l}x \ldots x \cdot x \ldots x \cdot x \ldots \\
x \ldots x \cdot x \cdot x \ldots x \ldots\end{array}$ & 3.7 & 92 & 3.6 & 90 & 4.0 & 100 \\
\hline$M$ & & & 3.2 & 77 & 3.5 & 84 & 3.8 & 94 \\
\hline
\end{tabular}

Note-The underlines indicate the transitions to better performance. *Locations of strong and weak accents. †The first rhythm of each pair is the upper one. ¥The pair number for pairs of different rhythms was assigned in terms of increasing discrimination.

son, the rhythms were presented at a slower tempo (roughly 2 notes $/ \mathrm{sec}$ ) and a faster tempo (roughly 3.5 notes $/ \mathrm{sec}$ ) to determine if the process of differentiation is indeed a function of tempo.

To summarize, the process of rhythm differentiation for nonmetric rhythms will be investigated by analyzing the changes in confusions across the differing alternation conditions. In addition, the rhythms will be presented at two different presentation rates to determine if differentiation is a function of tempo.

\section{METHOD}

\section{Subjects}

All 78 volunteers were undergraduates at the University of Tennessee, Knoxville, who received course credit for their participation. Roughly one half $(n=41)$ had no musical training at all, whereas the remaining subjects averaged 5.7 years of training. The subjects were run in small groups of one to three.

\section{Rhythms}

All rhythms, shown in Table 1, were based on five tones embedded in a repeating pattern of 16 units. The rules used to construct the patterns were: (1) a tone always occurred at the 1st unit; (2) a tone always occurred at the 12th unit and no tone occurred at Units 13,14,15, or 16; and (3) there was at least 1 silent unit, but no more than 3 silent units between any pair of tones. The combination of these three rules makes the longest silent interval ( 4 units long) occur at the end of the rhythm so that each rhythm would naturally be organized ending at that interval, according to the gap principle proposed by Garner and colleagues (Garner, 1974; Handel, 1974). Thus, the preferred organization of each rhythm begins at the lst unit, as displayed in Table 1. For every trial, all rhythms started at the 1st unit and ended at the 16th unit.

The above rules generate 16 possible patterns; 13 of these were used in the experiment. Three patterns, all beginning with a tone in Unit 1 and followed by three silent units, were eliminated merely to limit the number of possible rhythms. A total of 35 pairs of rhythms were constructed. Thirteen pairs consisted of two identi- cal rhythms, 1 pair for each of the rhythms. Twenty-two pairs contained two different rhythms. The pairs were selected from the 78 possible pairs to create a variety of differences. Specifically, the two rhythms in 3 pairs had the identical figural organization (Pairs 14-16 in Table 1; these 3 represent all the possible ones), the two rhythms in 10 pairs differed in the location of one tone (e.g., the fourth tone in Pair 18), whereas the two rhythms in 9 pairs differed in the location of two or three tones (e.g., the third and fourth tones in Pair 17). Using an alternative categorization, in 10 pairs the first difference occurred at the second tone (e.g., Pair 23), in 7 pairs the first difference occurred at the third tone (e.g., Pair 21), and in 5 pairs the first difference occurred at the fourth element (e.g., Pair 20). This set of 22 pairs was pretested in preliminary work to ensure that some pairs of rhythms would be very difficult to distinguish, whereas other pairs would be very easy to distinguish. Across the 22 different pairs, each rhythm was used three or more times (except for rhythm 1, which was used twice), and each rhythm occurred equally often as the first and second rhythm of the pair. Although the rhythms were always presented in the same order here, subsequent research has not shown any effect due to the order of the two rhythms.

\section{Task}

On every trial, two rhythms were presented and the subject judged whether the rhythms were the same or different, using a 4-point rating scale: $1=$ very sure identical, $2=$ fairly sure identical, $3=$ fairly sure different, and $4=$ very sure different. The first rhythm was always presented using a lower pitch tone $(400 \mathrm{~Hz}$, triangle waveform) and the second rhythm was always presented using a higher pitch tone (600 $\mathrm{Hz}$, triangle waveform).

\section{Alternation Conditions}

The rhythms were presented in six different ways, created by varying the number of alternations between the two rhythms and by varying the number of repetitions of one rhythm before it alternated to the other.

In three conditions, each rhythm was repeated only once. In the first, the two rhythms alternated one time (notated AX, to indicate that the presentation would be AA for pairs of identical rhythms and $\mathrm{AB}$ for pairs of different rhythms). Using Rhythms 1 and 2 as an example, the trial would be $x . x . x \ldots x \ldots x \ldots y \cdot y \ldots y \cdot y \ldots y \ldots$ 
In the second condition, the rhythms alternated two times (AXAX): $x \cdot x \cdot x \ldots x \ldots x \ldots y . y . y . y \ldots y \ldots . x . x . x \ldots x \ldots x \ldots y . y . y . y . . y \ldots$ In the third condition, the rhythms alternated four times (AXAXAXAX).

In two conditions, each rhythm was repeated twice. In the first, the two rhythms alternated one time (AAXX). Using the identical condition for Rhythm 1, the trial would be $\mathrm{x} . \mathrm{x} \cdot \mathrm{x} \ldots \mathrm{x} \ldots \mathrm{x} \ldots \mathrm{x} \cdot \mathrm{x} \cdot \mathrm{x} \ldots \mathrm{x} \ldots \mathrm{x} \ldots \mathrm{y} \cdot \mathrm{y} . \mathrm{y} . \mathrm{y} \ldots \mathrm{y} \ldots \mathrm{y} \cdot \mathrm{y} . \mathrm{y} . \mathrm{y} \ldots \mathrm{y} \ldots$. In the second condition, the rhythms alternated two times (AAXXAAXX).

In one condition, each rhythm was repeated four times and then the alternative rhythm was repeated four times (AAAAXXXX).

\section{Presentation Rates}

The rhythms were presented at two different rates. At the slower rate, the onset-to-onset interval between adjacent units was $167 \mathrm{msec}$ so that the length of one repetition was $2.67 \mathrm{sec}$ and there were 1.9 elements $/ \mathrm{sec}$. At the faster tempo, the onset-to-onset interval was $88 \mathrm{msec}$, so the length of one repetition was $1.4 \mathrm{sec}$ and there were 3.6 elements $/ \mathrm{sec}$. At both presentation rates, each tone was composed of a $10-\mathrm{msec}$ onset ramp, a $30-\mathrm{msec}$ steady state, and a 10 -msec offset ramp.

The rhythms were generated using BRS-Foringer modules; they were prerecorded and presented to the subjects using cassette tapes. The experimental session took place in a small room $(3 \times 4 \mathrm{~m})$ with acoustical ceiling tile. The subjects were seated $2.5 \mathrm{~m}$ from two speakers, stacked vertically, each of which presented the entire rhythm. The rhythms were presented at a comfortable listening level, approximately $65 \mathrm{~dB}$ SPL (the subjects were allowed to adjust the loudness if they wished).

\section{Experimental Design}

There were 12 conditions, obtained by crossing the 6 alternation conditions with the two presentation rates. These 12 conditions were broken into three blocks of 4 conditions. Within each block, 4 different alternation conditions were used; 2 conditions were at the faster rate and 2 were at the slower rate. To minimize any effect due to the particular conditions presented within a block, or the order within a block, there were three different ways that the 12 conditions were broken into three blocks of 4 conditions apiece. The order of presentation of the conditions within a block were counterbalanced.

Each subject was presented with one block of four conditions. Each condition consisted of 35 rhythm pairs, 13 identical pairs, and 22 different pairs, presented in random orders. Two strategies were used to acquaint the subject with each condition. First, before the actual presentation, four practice trials were given, using simple four-tone rhythms and the same condition. Two of the trials consisted of identical pairs and two consisted of different pairs. If the subjects were confused, then these patterns were repeated until they felt confident. Second, the first 2 rhythm pairs were repeated later among the 35 experimental trials (thus, there were 37 trials per condition). The subjects were not told that these were practice trials and the results were not utilized. The subjects did not receive any feedback about their performance. There were short breaks between the four conditions so that the experimental session lasted about $75 \mathrm{~min}$.

The results for each of the 12 conditions are based on a minimum of 25 different subjects and a maximum of 27 different subjects. This variation was due to different numbers of subjects at an experimental session.

\section{RESULTS}

The data were summarized using two measures. The first measure was the average rating for each rhythm pair. Better performance for the identical pairs would result in average ratings between 1 and 2; better performance for the different pairs would result in average ratings between 3 and 4 . The second measure was the percentage correct, which was obtained by combining the 1 rating (very sure identical) and 2 rating (fairly sure identical) for the identical pairs, and by combining the 3 rating (fairly sure different) and 4 rating (very sure different) for the different pairs. Although these measures are obviously dependent, each can provide slightly different views of the outcomes.

Preliminary analyses indicated that there were no effects due to the four positions within one block presented to a subject. For the four positions, the percentages correct for the slow presentation rate were $68 \%, 71 \%, 68 \%$, and $70 \%$ and the percentages correct for the fast presentation rate were $65 \%, 69 \%, 68 \%$, and $65 \%$. Thus, there was no evidence of either leaming or fatigue effects. There were, however, large individual differences that will be discussed later in this section, but because these did not interact with the other variables, the data were averaged across all subjects.

The data were initially analyzed using a 2 (rate) $\times 6$ (alternation condition) $\times 22$ (pattern) analysis of variance (ANOVA) with pattern as the sampling variable. The results indicated that these were significant main effects for alternation condition $[F(5,105)=15.9, p<.001]$ and pattern $[F(21,105)=65.9, p<.001]$, as well as a significant interaction for rate $\times$ alternation condition $[F(5,105)$ $=14.5, p<.001]$. Subsequent two-way ANOVAs at each rate indicated significant differences between alternation conditions at both the slow rate $[F(5,105)=23.0, p<$ $.001]$ and the fast rate $[F(5,105)=8.8, p<.001]$. Due to the interactions with rate of presentation, the following results are organized at the first level in terms of rate. At each presentation rate, the judgments are analyzed in terms of alternation condition and then rhythm pair.

\section{Slow Presentation Rate (1.9 Elements/Sec)}

The results for the slow presentation rate are shown in Table 1. For the identical pairs, there was little difference among the rhythm pairs or the alternation conditions. In contrast, for the different rhythm pairs, there were large differences among the rhythm pairs and the alternation conditions, so the summary procedures detailed below were based on the performance for the different pairs.

The first step was to consider the six alternation conditions. These six conditions divided naturally into three groups, based on a posteriori pairwise comparisons. The first group consisted of the AX and AXAX conditions, which generated the lowest average rating (percent correct): 2.7 (57\% correct) and $2.9(62 \%)$, respectively. There were no consistent differences between the two. Of the six conditions, these two yielded the two lowest average ratings and percent correct for 15 of the 22 different pairs. The second group consisted of the AXAXAXAX condition, which generated the best performance: $3.42(82 \%)$. Of the six conditions, this one yielded the highest average 
rating and percent correct for 18 of the 22 different pairs. The third group consisted of the AAXX, AAXXAAXX, and AAAAXXXX conditions, which generated intermediate performance: $3.02(70 \%), 3.12(71 \%)$, and 3.12 (73\%) respectively. There were no consistent differences among the conditions. On this basis, the results for the $\mathrm{AX}$ and AXAX conditions were combined and the results from the AAXX, AAXXAAXX, and AAAAXXXX conditions were combined.

Identical pairs. The results for the pairs of identical rhythms are shown in the top part of Table 1. Overall, performance was very good. Performance was slightly better for the AXAXAXAX condition, but the differences among alternation conditions were small and unimportant. Similarly, performance was nearly the same for all identical pairs. With the exception of the identical pair constructed from Rhythm 3 (x.x..x...x.x...), the percentage correct for each pair averaged about $90 \%$. The reason for the poorer performance for this rhythm is unknown, but probably reflects only chance variation.

Different pairs. The outcomes for the pairs constructed from different rhythms is shown in the bottom half of Table 1 . The rhythm pairs were placed into four groups, based on the average ratings and percent correct on the three categories of alternation conditions.

The first group consisted of three pairs that tended to be perceived as being identical for all alternation conditions. Even the best performance was at less-than-chance level; the majority of judgments $(55 \%)$ were 1 (very sure identical). For these pairs, discrimination was only slightly poorer at the most difficult conditions (AX and AXAX). However, the discrimination for these pairs was not simply equivalent to that for identical pairs; the subjects judged these as more likely to be different than the identical pairs.

What characterizes these pairs is that the figural or element grouping description is the same for each rhythm. Moreover, the accents, according to the rules suggested by Povel and Okkerman (1981) also are identical. Perceptually, tones separated by one silent space are heard as forming groups, and tones separated by two or three silent spaces are heard as being in different groups. Listeners perceive all of the silent intervals as being equal and they are unable to make use of the differences in timing between the rhythmic groups, even though the differences in the onset intervals are way beyond the estimated difference threshold of $10 \%: 500 \mathrm{msec}(\mathrm{x} . \mathrm{x})$ to $664 \mathrm{msec}(\mathrm{x}$. . . x) (see Hirsh, Monahan, Grant, \& Singh, 1990). Therefore, for the first pair, the description for each rhythm would be three elements, space, one element, space, one element, space (i.e., 3-1-1). For the second pair, the description for each rhythm would be two elements, space, two elements, space, one element, space (i.e., 2-2-1), whereas for the third pair, the description of each rhythm would be two elements, space, one element, space, two elements, space (i.e., 2-1-2). We would expect that any pair of rhythms with the same pattern of groups would be judged as being identical. For example, in subsequent research, the pair $x \ldots x . x . x \ldots x \ldots$ (i.e., $1-3-1)$ and $x$. . x.x.x ... . . . (i.e., 1-3-1) was judged as being identical.

The second group of four pairs was characterized by poor performance for the most difficult five alternation conditions, but relatively good performance for the easiest condition. (Poor performance is defined as an average rating below 3.0, combined with a percent correct below $70 \%$.) All four of these pairs possess two salient timing characteristics. First, the timing and resulting grouping of the initial two or three tones is identical in both rhythms. Therefore, the discrimination between the two rhythms must be made on the basis of the final elements. Second, the final elements for one of the two rhythms are equally spaced, whereas the final elements for the other rhythm are not. The clearest example illustrating these two factors is Pair 18. For this pair, the timing is identical for the first three tones and the only difference between the rhythms is the placement of the fourth tone. For Rhythm 8 , the fourth tone is equally spaced between the surrounding tones so that the element grouping is $1-2-1-1$, but for Rhythm 12 , the fourth tone is normally grouped with the final tone so that the element grouping is $1-2-2$.

What this suggests is that without the ability to compare the rhythms created by the AXAXAXAX alternation condition several times, the subjects made their judgments on the basis of the identical timing and grouping of the initial tones because they found it difficult to distinguish between the slightly different element grouping at the end of the pattern.

The third group of six pairs was characterized by poor discrimination at the two most difficult alternation conditions, relatively good discrimination at the three intermediate conditions, and excellent discrimination at the easiest alternation condition. For these pairs, the timing of the initial elements differs (except for Pair 25), although there are segments of the rhythm that have the identical timings. For example, consider Pair 26. In this case, even though the initial tones vary, there is a group of two tones in the middle that are identical. Rhythm 2 would be coded 2-2-1, whereas Rhythm 9 would be coded 1-1-2-1. It is this gross similarity that makes it difficult to discriminate between the two rhythms, given only one or two alternations of a single cycle of each rhythm.

The fourth group of nine pairs was relatively easy to discriminate at all alternation conditions. Moreover, these pairs were easier to discriminate even at the easiest alternation condition. Within this group, five pairs contain one or more rhythms that contain three adjacent tones, forming a perceptual group of three (Pairs 27, 28, 29, 34, and 35 ). It is this run of three elements that probably made those rhythms easy to distinguish. The remaining fours pairs are a mixed bag, and some of the results are surprising. It would seem that Pair 33 would be more difficult to distinguish, due to the similarity between the element grouping of the two rhythms (e.g., 2-2-1 for Rhythm 5; 1-1-2-1 for Rhythm 9). 
Summary. These analyses reinforce the view that listeners perceive rhythms in terms of grouping of the elements and are unable to make use of the differences in timings between groups or runs of tones. For those rhythm pairs in which the grouping structure is identical (Pairs 1416), performance was below chance for all alternation conditions. For those rhythm pairs in which the grouping structure differs (Pairs 17-35), increasing the number of repetitions of each rhythm and/or increasing the number of alternations between rhythms improved performance up to a point. On the whole, the judgments seemed to be based more on the grouping of the initial two or three tones, rather than on the grouping of the last two or three tones.

It is interesting that the subjects rarely judged pairs of identical rhythms as being different. This may reflect a simple, context-independent response bias toward judging rhythms as being the same. Alternately, it may reflect perceptual differentiation in which a global, diffuse stimulus becomes progressively structured. Until this differentiation occurs, subjects cannot perceive timing differences between the two rhythms. Thus, the preponderance of "identical" responses is a consequence of the process of perceptual differentiation.

\section{Fast Presentation Rate (3.6 Elements/Sec)}

The results for the fast presentation rate are shown in Table 2. As was found at the slow presentation rate, there were only small differences for the pairs of identical rhythms. Thus, the summary procedures described below are based on the performance for the pairs of different rhythms.

The first step was to place the alternation conditions into groups. Three groups emerged from a posteriori pairwise comparisons. The first group consisted of the $\mathrm{AX}$ condition, which yielded the lowest average rating (percent correct): $2.8(60 \%)$. Of the six conditions, this one yielded the lowest values for 11 of the 22 different rhythm pairs. The second group consisted of the AAXXAAXX condition, which generated the best performance: $3.3(78 \%)$. Of the six conditions, this one yielded the highest values for 17 of the 22 different rhythm pairs. The third group, AAXX, AAAAXXXX, AXAX, and AXAXAXAX, produced intermediate levels of performance. For the different rhythm pairs, the average ratings and average percent correct for these conditions were $3.0(65 \%), 3.0$ $(67 \%), 3.1(70 \%)$, and $3.0(66 \%)$, respectively. The results for the identical and different pairs will be analyzed separately below.

Identical pairs. The results for the identical pairs are shown in the top portion of Table 2. Overall, performance was excellent; the percent correct averaged well over $90 \%$. The AAXXAAXX alternation condition produced slightly better performance: for 6 of the 13 pairs, the average rating and percent correct was the highest among all six conditions, although any single difference was small. These results are therefore identical to those found at the slow rate.
Different rhythm pairs. The results for the different pairs are shown in the bottom section of Table 2. As was done for the slow presentation rate, the pairs were placed into groups based on the average ratings and percent correct across the alternation conditions. Four groups emerged.

1. The first group consisted of three pairs that tended to be perceived as identical at all alternation conditions. Although the subjects judged these pairs of rhythms as more likely to be different than pairs of identical rhythms, performance was at less-than-chance level (i.e., 50\% correct). The majority of judgments $(62 \%)$ were 1 (very sure identical). These are the same three pairs that were found to be identified at less than chance for the slow presentation rate. As argued previously, each pair of rhythms has the identical figural organization, and listeners were unable to use the differences among the silent intervals between the final and initial tones in successive groups to distinguish between the rhythms.

2 . The second group of six pairs was characterized by poor performance and relatively large differences among pairs at the most difficult (AX) condition, slightly better performance with minimal differences among patterns at the four intermediate conditions, and good performance (i.e., mean rating above 3 ) at the easiest (AAXXAAXX) condition. These patterns tend to be characterized by differences in element groupings at the beginning of the rhythms, but identical groupings of elements at the end of the rhythms. This can be seen most easily for Pairs 24, 32 , and 33, which had the poorest discrimination. For Pair 24, Rhythm 12 would be organized as $1-2-2$, whereas Rhythm 3 would be organized as $1-1-1-2$. The rhythms in Pairs 32 and 33 are even more similar. For Pair 33, the organization of Rhythms 5 and 9 would be 2-2-1 and 1-1-2-1, respectively, and for Pair 32, the organization of Rhythms 10 and 6 would be $1-1-1-2$ and $2-1-2$, respectively. The similarity in timing and grouping for the other three pairs is not as obvious. In particular, the discrimination for Pair 31 should have been better, given the differences in grouping: Rhythm 9 would be 1-1-2-1, whereas Rhythm 7 would be $2-1-1-1$.

3 . The third group of four pairs was distinguished by poor performance at the most difficult condition, reasonably good performance at the intermediate conditions, and excellent performance at the easiest condition. These pairs of rhythms tend to be characterized by the fact that the timing of the last two tones is identical. Thus, these pairs are similar to the second group, discussed above, in that the similarity of timing of the final elements led the subjects to incorrectly perceive that the two rhythms were identical for the AX condition. These rhythm pairs tend to be more dissimilar in terms of the overall pattern of timings and groupings, and it is probably this factor that yielded better performance at the intermediate alternation conditions.

4. The fourth group of nine pairs was distinguished by excellent performance at all the alternation conditions. In fact, there were no differences among the six conditions. 
Table 2

Average Ratings (AR) and Percent Correct (PC) for all Pairs of Rhythms at the Fast (3.6 Elements/Sec) Presentation Rate

\begin{tabular}{|c|c|c|c|c|c|c|c|c|}
\hline \multirow{3}{*}{$\begin{array}{c}\text { Pair } \neq \\
\text { Number }\end{array}$} & \multirow[b]{3}{*}{ Rhythm $†$} & \multirow{3}{*}{$\begin{array}{c}\text { Rhythms } \\
\text { Timing }\end{array}$} & \multicolumn{6}{|c|}{ Alternation Conditions } \\
\hline & & & \multicolumn{4}{|c|}{$\begin{array}{c}\text { AXAX } \\
\text { AXAXAXAX } \\
\text { AAXX } \\
\text { AAAAXXXX }\end{array}$} & \multicolumn{2}{|c|}{ AAXXAAXX } \\
\hline & & & AR & PC & AR & PC & AR & PC \\
\hline
\end{tabular}

Identical Rhythms

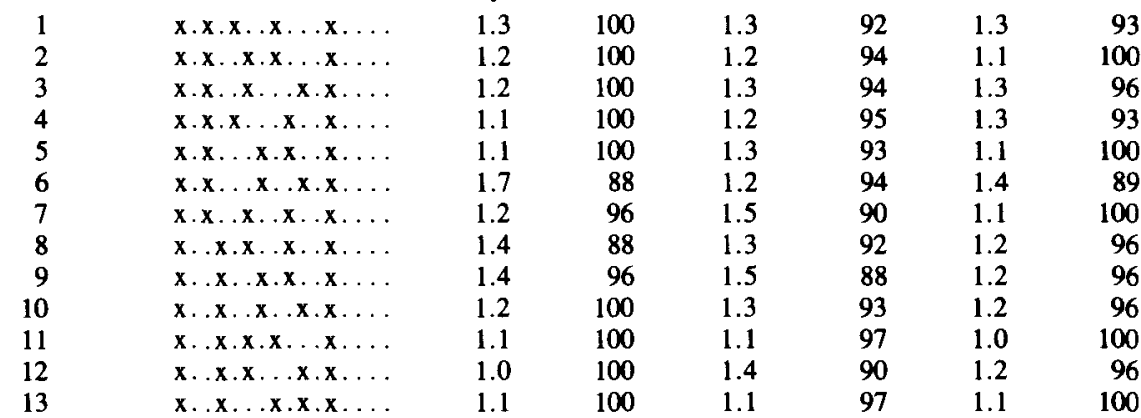

Different Rhythms

\begin{tabular}{|c|c|c|c|c|c|c|c|c|}
\hline 14 & 1 & $\mathbf{x} \cdot \mathbf{x} \cdot \mathbf{x} \ldots \mathbf{x} \ldots \mathbf{x} \ldots$ & 1.4 & 8 & 1.6 & 15 & 1.3 & 15 \\
\hline & 4 & $\mathbf{x} \cdot \mathbf{x} \cdot \mathbf{x} \ldots \mathbf{x} \ldots \mathbf{x} \ldots$ & & & & & & \\
\hline 15 & 2 & $x \cdot x, x, x \ldots x \ldots$ & 1.5 & 15 & 1.9 & 26 & 1.8 & 26 \\
\hline \multirow[t]{2}{*}{16} & $\begin{array}{l}5 \\
6\end{array}$ & $\begin{array}{c}x \cdot x \ldots x \cdot x \ldots x \ldots \\
x \cdot x \ldots x \ldots x \cdot x \ldots\end{array}$ & 1.6 & 15 & 1.6 & 14 & 1.5 & \\
\hline & 3 & $\mathbf{x} \cdot \mathbf{x} \ldots \mathbf{x} \ldots \mathbf{x}, \mathbf{x} \ldots$ & & & & & & \\
\hline \multicolumn{2}{|l|}{$M$} & & 1.5 & 13 & 1.7 & 18 & 1.5 & 19 \\
\hline \multirow[t]{2}{*}{24} & 12 & $x \ldots x \cdot x \ldots x \cdot x \ldots$ & 1.9 & 27 & 2.9 & 55 & 3.4 & 85 \\
\hline & 3 & $x \cdot x \ldots x \ldots x \cdot x \ldots$ & & & & & & \\
\hline \multirow[t]{2}{*}{33} & 5 & $\mathbf{x} \cdot \mathbf{x} \ldots \mathbf{x} \cdot \mathbf{x} \ldots \mathbf{x} \ldots$ & 2.3 & 38 & 2.9 & 69 & 3.2 & 78 \\
\hline & 9 & $x \ldots x \ldots x \cdot x \ldots x \ldots$ & & & & & & \\
\hline \multirow[t]{2}{*}{32} & 10 & $x \ldots x \ldots x, x, x \ldots$ & 2.7 & 54 & 2.9 & 65 & 3.4 & 89 \\
\hline & 6 & $\mathbf{x} \cdot \mathbf{x} \ldots \mathbf{x} \ldots \mathbf{x} \cdot \mathbf{x} \ldots$ & & & & & & \\
\hline \multirow[t]{2}{*}{31} & 9 & $\mathbf{x} \ldots \mathbf{x} \ldots \mathbf{x} \cdot \mathbf{x} \ldots \mathbf{x} \ldots$ & 2.7 & 57 & 2.8 & 62 & 3.4 & 78 \\
\hline & 7 & $\mathbf{x} \cdot \mathbf{x} \ldots \mathbf{x} \ldots \mathbf{x} \ldots \mathbf{x} \ldots$ & & & & & & \\
\hline \multirow[t]{2}{*}{26} & 2 & $x \cdot x \ldots x \cdot x \ldots x \ldots$ & 2.8 & 57 & 2.9 & 64 & 3.7 & 89 \\
\hline & 9 & $x \ldots x \ldots x \cdot x \ldots x \ldots$ & & & & & & \\
\hline \multirow[t]{2}{*}{18} & 8 & $\mathbf{x} \ldots \mathbf{x} \cdot \mathbf{x} \ldots \mathbf{x} \ldots \mathbf{x} \ldots$ & 2.9 & 65 & 2.8 & 61 & 3.3 & 81 \\
\hline & 12 & $\mathbf{x} \ldots \mathbf{x} \cdot \mathbf{x} \ldots \mathbf{x} \cdot \mathbf{x} \ldots$ & & & & & & \\
\hline$M$ & & & 2.6 & 50 & 2.9 & 64 & 3.4 & 83 \\
\hline \multirow[t]{2}{*}{29} & 4 & $x \cdot x \cdot x \ldots x \ldots x \ldots$ & 2.7 & 57 & 3.3 & 78 & 3.7 & 89 \\
\hline & 8 & $x \ldots x \cdot x \ldots x \ldots x \ldots$ & & & & & & \\
\hline \multirow[t]{2}{*}{23} & 13 & $\mathbf{x} \ldots \mathrm{x} \ldots \mathrm{x} \cdot \mathrm{x} \cdot \mathrm{x} \ldots$ & 2.8 & 62 & 3.3 & 78 & 3.7 & 93 \\
\hline & 6 & $\mathbf{x} \cdot \mathbf{x} \ldots \mathbf{x} \ldots \mathbf{x}, \mathbf{x}, \ldots$ & & & & & & \\
\hline \multirow[t]{2}{*}{28} & 11 & $x \ldots x \cdot x \cdot x \ldots x \ldots$ & 3.1 & 62 & 3.2 & 79 & 3.7 & 96 \\
\hline & 2 & $x \cdot x \ldots x \cdot x \ldots x \ldots$ & & & & & & \\
\hline \multirow[t]{2}{*}{19} & 8 & $\mathbf{x} \ldots \mathbf{x} \cdot \mathbf{x} \ldots \mathbf{x} \ldots \mathbf{x} \ldots$ & 3.1 & 65 & 3.1 & 75 & 3.5 & 85 \\
\hline & 9 & $\mathbf{x} \ldots \mathbf{x} \ldots \mathbf{x}, \mathbf{x} \ldots \mathbf{x} \ldots$ & & & & & & \\
\hline$M$ & & & 2.9 & 62 & 3.2 & 78 & 3.7 & 91 \\
\hline \multirow[t]{2}{*}{21} & 10 & $x \ldots x \ldots x \ldots x \cdot x \ldots$ & 3.1 & 77 & 3.1 & 71 & 3.8 & 96 \\
\hline & 8 & $\mathbf{x} \ldots \mathbf{x} \cdot \mathbf{x} \ldots \mathbf{x} \ldots \mathbf{x} \ldots$ & & & & & & \\
\hline \multirow[t]{2}{*}{17} & 6 & $\mathbf{x} \cdot \mathbf{x} \ldots \mathbf{x} \ldots \mathbf{x}, \mathbf{x} \ldots$ & 3.2 & 77 & 3.0 & 70 & 3.4 & 78 \\
\hline & 7 & $\mathbf{x}, \mathbf{x} \ldots \mathbf{x} \ldots \mathbf{x} \ldots \mathbf{x} \ldots$ & & & & & & \\
\hline \multirow[t]{2}{*}{30} & 9 & $x \ldots x \ldots x \cdot x \ldots x \ldots$ & 3.2 & 77 & 3.2 & 75 & 3.6 & 85 \\
\hline & 10 & $\mathbf{x} \ldots \mathbf{x} \ldots \mathbf{x} \ldots \mathbf{x} \cdot \mathbf{x} \ldots$ & & & & & & \\
\hline
\end{tabular}


Table 2 (Continued)

\begin{tabular}{|c|c|c|c|c|c|c|c|c|}
\hline \multirow{3}{*}{$\begin{array}{c}\text { Pair } \ddagger \\
\text { Number }\end{array}$} & \multirow[b]{3}{*}{ Rhythm $\dagger$} & \multirow{3}{*}{$\begin{array}{c}\text { Rhythms } \\
\text { Timing }\end{array}$} & \multicolumn{6}{|c|}{ Alternation Conditions } \\
\hline & & & \multicolumn{2}{|c|}{$\mathrm{AX}$} & \multicolumn{2}{|c|}{$\begin{array}{c}\text { AXAX } \\
\text { AXAXAXAX } \\
\text { AAXX } \\
\text { AAAAXXXX }\end{array}$} & \multicolumn{2}{|c|}{ AAXXAAXX } \\
\hline & & & AR & PC & $\mathbf{A R}$ & $\mathrm{PC}$ & AR & PC \\
\hline \multirow[t]{2}{*}{34} & 3 & $x \cdot x \ldots x \ldots x \cdot x \ldots$ & 3.3 & 85 & 3.5 & 86 & 3.7 & 93 \\
\hline & 4 & $x \cdot x \cdot x \ldots x \ldots x \ldots$ & & & & & & \\
\hline \multirow[t]{2}{*}{20} & 7 & $\mathbf{x} \cdot \mathbf{x} \ldots \mathbf{x} \ldots \mathbf{x} \ldots$ & 3.4 & 81 & 3.4 & 84 & 3.7 & 93 \\
\hline & 2 & $\mathbf{x} \cdot \mathbf{x} \ldots \mathbf{x} \cdot \mathbf{x} \ldots \mathbf{x} \ldots$ & & & & & & \\
\hline \multirow[t]{2}{*}{27} & 11 & $x \ldots x \cdot x \cdot x \ldots x \ldots$ & 3.4 & 88 & 3.3 & 76 & 3.3 & 78 \\
\hline & 1 & $\mathbf{x} \cdot \mathbf{x} \cdot \mathbf{x} \ldots \mathbf{x} \ldots \mathbf{x} \ldots$ & & & & & & \\
\hline \multirow[t]{2}{*}{22} & 13 & $x \ldots x \ldots x \cdot x \cdot x \ldots$ & 3.4 & 91 & 3.4 & 81 & 3.8 & 96 \\
\hline & 5 & $x, x \ldots x, x \ldots x \ldots$ & & & & & & \\
\hline \multirow[t]{2}{*}{25} & 9 & $x \ldots x \ldots x . x \ldots$ & 3.5 & 91 & 3.1 & 74 & 3.4 & 85 \\
\hline & 13 & $\mathbf{x} \ldots \mathbf{x} \ldots \mathbf{x} \cdot \mathbf{x} \cdot \mathbf{x} \ldots$ & & & & & & \\
\hline \multirow[t]{2}{*}{35} & 12 & $\mathbf{x} \ldots \mathbf{x} \cdot \mathbf{x} \ldots \mathbf{x} \cdot \mathbf{x} \ldots$ & 3.8 & 91 & 3.6 & 89 & 3.8 & 93 \\
\hline & 11 & $x \ldots x \cdot x \cdot x \ldots x \ldots$ & & & & & & \\
\hline$M$ & & & 3.4 & 84 & 3.3 & 78 & 3.6 & 89 \\
\hline
\end{tabular}

Note-The underlines indicate the transitions to better performance. *Locations of strong and weak accents. †The first rhythm of each pair is the upper one. ¥The pair numbers are those assigned for the slow presentation rate.

What characterizes these pairs of patterns is that the timing and grouping of the final pair of tones differs between the two rhythms. The only pair that does not fit this generalization is Pair 27, and here the difference between the two rhythms can easily be heard, due to the runs of three elements.

Summary. These results reinforce the conclusions that listeners perceive nonmetric rhythms in terms of the groupings of the tones and not in terms of the timings between the groups. As was found at the slow presentation rate, for those pairs in which the grouping structure of each rhythm was identical, performance was below chance at every condition. For pairs with different groupings, increasing the number of repetitions and/or alternations improved discrimination. On the whole, the pattern of outcomes suggests that rhythm similarity was based mainly on the timings and groupings at the end of the rhythm. Finally, the subjects rarely judged identical pairs as being different.

\section{Individual Differences}

There were large individual differences in the ability of the subjects to discriminate between two rhythms. One simple way to demonstrate this is to divide the subjects in each alternation condition in half on the basis of the percent correct for the pairs of different rhythms. Because each subject was given four conditions, the number of times each subject performed above the median could range from 4 to 0 . Across the 78 subjects, $17(22 \%)$ were above the median for all four conditions, and $19(24 \%)$ were below the median for all four conditions. These values should be compared to expected values of $9.75(1 / 8$ or $12.5 \%$ ) if performance was independent between conditions. The remaining subjects were distributed fairly equally: 3 above/ 1 below median $(n=16), 2$ above $/ 2$ below $(n=13)$, and 1 above/ 3 below $(n=13)$. Thus, the subjects clearly were not distributed according to binomial expected values-either they were able to discriminate the rhythms across a wide range of conditions and presentation rates, or they performed poorly across the same variety of conditions.

The individual differences can be illustrated more concretely by considering the two most difficult conditions for the slow presentation rate, AX and AXAX. For the three most difficult pairs $(14,15$, and 16$)$, there were no differences between subjects above the median or below the median. For these pairs, even the best subjects were unable to make use of the timing differences between the longer silent intervals separating the groups of elements in order to judge that the two rhythms were different. However, there were large and consistent differences for all of the other pairs of rhythms. First, consider the second group of patterns (average rating was $2.7,60 \%$ correct). For the subjects above the median, the average rating was 3.1 with $74 \%$ correct, but for the subjects below the median, the average rating was 2.4 with $44 \%$ correct. Next, consider the third group of pairs of rhythms (average rating of $2.9,62 \%$ correct). For the subjects above the median, performance improved to an average rating of 3.5 with $85 \%$ correct, but for the subjects below the median, performance remained the same, an average rating of 2.3 with $38 \%$ correct. Finally, consider the fourth group of pairs of rhythms (average rating of 3.2, $77 \%$ correct). For the subjects above the median, performance was nearly perfect, with an average rating of 3.7 and $92 \%$ correct. For the subjects below the median, the improvement in performance was small; the average rating was only 2.7 with $60 \%$ correct. These comparisons point out that the individual differences between subjects emerge most strongly for the easier pairs of different rhythms. For the most difficult pairs, where the figural grouping was equivalent, there was no difference. But as 
the timing of the two rhythms became more different, so that the figural grouping differed, the performance of the better subjects increased rapidly, whereas the performance of the poorer subjects increased only modestly.

\section{DISCUSSION}

\section{Figural Rhythmic Perception}

The nonmetric rhythms used here were perceived as groups, or runs, of tones and the timing between the groups was perceived poorly. One might argue that these rhythms were perceived in terms of only two intervals: a short interval that served to group the tones and a longer interval that served to separate the groups. For these rhythms, the different-length intervals that separate the groups are assimilated to one value so that differences between these intervals cannot be used to distinguish between the rhythms.

It is difficult to hear the differences between the rhythms that have the identical figural organizations in Pairs 14, 15 , and 16 , even if the key intervals are known beforehand. Each of these six rhythms contains a middle group of one element (Rhythms 1, 3, 4, and 6) or two elements (Rhythms 2 and 5) that "floats." There are two units of silence on one side and three units of silence on the other side. Thus, a shift of the middle element(s), such that the two and three units of silence reverse, does not change the figural grouping. The number of identical elements between two rhythms does not predict the ability to distinguish between two rhythms. For example, consider Pair 15, which is composed of Rhythms 2 and 5 . In this case, there are two differences, Elements 3 and 4, between the rhythms. Now consider Pair 28, which is composed of Rhythms 2 and 11 . Here there is only one difference, Element 2, and yet performance is very good. In subsequent work, the two rhythms $x \ldots x . x . x \ldots x \ldots$ and $x \ldots x . x . x \ldots x$ were identified at a less-than-chance level because the grouping was identical (1-3-1), even though the position of all three middle tones differed. Thus, timing differences per se do not predict difficulty, but do so only to the extent that they affect grouping.

Similar results have been found by Monahan and Hirsh (1990). In that work, subjects discriminated between sixtone metric rhythmic patterns in which the onset of one tone could be delayed. They found that the poorest discrimination performance of the delayed tone occurred between two long silent intervals. For example, the poorest performance occurred for the capitalized tone in the fol-

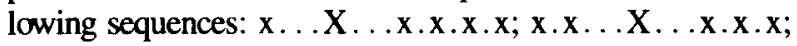
and $x . X . X \ldots X \ldots x . x$. In our conceptualization, the capitalized tone "floats" between groups. The subjects were unable to make fine timing distinctions for intervals between groups unless the timing change resulted in a different grouping. In these cases, small delays do not affect the grouping, so the magnitude of the delay must be increased before the rhythms become perceptually different. This outcome reinforces the above conclusion: the same timing change may have vastly different effects, depending on the organization of the rhythm.

\section{Differentiation of Rhythmic Structure}

The perception of these rhythms does undergo differentiation as the rhythms are recycled and/or alternated. At the most difficult condition, with rare exceptions, the subjects were only able to discriminate between two rhythms if one contained a run of three elements; we can argue that it is this feature that constitutes the figural percept. At the intermediate conditions, in which each rhythm was repeated or alternated several times, the subjects obtained a more differentiated percept. Even so, the limitations on the number of repetitions or the number of alternations precludes the development of an accurate figural description of the grouping of the tones. The subjects still perceived different rhythms as being identical; the rhythm was not structured completely. Finally, at the easiest repetition condition, the subjects appeared to have created a complete figural description. Thus, except for those pairs of rhythms with the identical descriptions, discrimination was nearly perfect. Thus, the progression goes from picking up one salient discriminating feature (e.g., a grouping of three tones), to picking up the grouping in one part of the rhythm, to picking up the grouping in the entire rhythm.

It is clear that several factors limit the ultimate level of the perceived rhythmic structure. First, the alternation conditions can limit the level by not giving listeners enough opportunities to apprehend the timings and groupings. Second, the nature of the rhythm itself can determine the level. All of the patterns used here were nonmetric, according to musical definitions, and it is possible that without the schema imposed by a meter, listeners cannot make fine-grained temporal discriminations. This issue is still unresolved. In spite of several studies on manual timing (Essens \& Povel, 1985), I am not convinced that listeners cannot accurately perceive nonmetric rhythms. Third, the presentation rate, or tempo, can determine the level. Both very slow and very rapid rates can change the tonal grouping (see Handel, 1989) and changes in rate, even with the identical grouping, can result in differences in the "goodness" of the rhythm. These rhythms sound best, according to casual questioning of listeners, at the slower presentation rate. For some reason, the rhythms lose their syncopated "feel" at the fast rate.

It is unclear how to assess the role of metric accents in the results. The rhythms are 16 elements long, so that the most important metric accent would occur on Elements 1 and 9 and the minor accents would occur on Elements 5 and 13. It could be hypothesized that pairs of rhythms with metric accents should be easy to discriminate because the accents induce a time frame to more easily perceive any differences between two similar rhythms (see Bharucha \& Pryor, 1986). The results here do not support such a hypothesis. Rhythm 4 has tones at Elements 1, 5, and 9. (We are assuming, following Povel \& Essens, 1985 , that the metric accent is strongest if a tone occurs at those time points.) This rhythm cannot be discriminated from Rhythm 1, which has no metric accents, but is discriminated from Rhythm 13 , which also has no metric accents. Discrimination is intermediate between Rhythm 4 
and 9 , which both have a tone at Element 9 . Thus, for this rhythm pair, as for the others, discrimination is not a function of the number of metric accents or the number of shared accents. It still is an open question, of course, whether the differentiation of timing for metric rhythms follows the same sequence as that found for nonmetric rhythms.

\section{Presentation Rate}

There are two outcomes that suggest that these rhythms are perceived differently at the slow and fast rates. First, the relative difficulty of the alternation conditions was not the same. Although the poorest discrimination for both rates was $A X$, the best discrimination for the slow rate occurred for AXAXAXAX, but the best discrimination for the fast rate occurred for AAXXAAXX. Furthermore, there is a different pattern of difficulty among the alternation conditions at the two rates. At the slow rate, the $A X$ and AXAX conditions and the AAXX and AAXXAAXX conditions yielded equal discrimination, even though it would appear that the extra repetitions ought to yield superior performance. At the fast rate, however, AXAX and AAXXAAXX yielded better discrimination than AX and AAXX did, respectively. The same paradox occurred for AXAX and AXAXAXAX; there was no difference in discrimination at the fast rate, but there was a large difference at the slow rate. It is unclear whether these outcomes have implications for a general theory of rhythm or whether they merely reflect the particulars of this task. In other words, the subjects found it difficult to rapidly switch back and forth between the two rhythms at the faster rate so that they could attend better if the rhythm was repeated twice before alternating.

Second, the relative difficulty of pairs of different rhythms varied between the two rates. Although there were exceptions, at the slow rate, it seems that the subjects made their discriminations based on the timing and grouping of the initial elements, whereas at the fast rate, it seems that they made their discriminations based on the timing and grouping of the final elements. These differences can easily be seen by considering those pairs of different patterns that dramatically changed their relative ranking at the two rates. Pairs 17 and 20 were difficult at the slow rate, but easy at the fast rate. These two pairs began with the identical figural grouping. Pairs 32 and 33 were easy at the slow rate, but difficult at the fast rate. These two pairs ended with the identical figural grouping. The rhythms were probably perceived differently at the two rates. After all, the interval between the onset of tones in a group at the slow rate $(332 \mathrm{msec})$ was equivalent or greater than the interval between the onset of two tones in different groups at the fast rate (e.g., x. . x equals $264 \mathrm{msec}$, and $x$... x equals $352 \mathrm{msec}$ ). At this point however, it is difficult to operationalize any differences.

\section{Summary}

The finding that discrimination improves in a logical way as the number of repetitions and/or alternations of the rhythms increases makes it clear that the general principles suggested by Krueger (see Sander, 1930) and Werner (Flavell \& Dragons, 1957; Smith, 1957) underlie the differentiation of auditory rhythmic structure. There is an initial figure-ground structuring in which tones are grouped into runs and then a gradual unfolding of the timing and grouping structure. The perception progresses from being more regular and uniform to being more articulated and irregular. As is true for all modalities and contexts, general constraints act to limit the ultimate level of the differentiation of the structure.

\section{REFERENCES}

Bamberger, J. (1978). Intuitive and formal musical knowing: Parables of cognitive dissonance. In S. S. Madeja (Ed.), The arts, cognition and basic skills (pp. 173-209). St Louis: CEMREL.

Bharucha, J. J., \& Pryor, J. H. (1986). Disrupting the isochrony underlying rhythm: An asymmetry in discrimination. Perception \& Psychophysics, 40, 137-141.

COOPER, G., MEYER, L. B. (1960). The rhythmic structure of music. Chicago: University of Chicago Press.

Essens, P. J., \& PoVEL, D. -J. (1985). Metrical and nonmetrical representations of temporal patterns. Perception \& Psychophysics, 37, 1-7.

Flavell, J. H., \& Draguns, J. (1957). A microgenetic approach to perception and thought. Psychological Bulletin, 54, 197-217.

Fraisse, P. (1982). Rhythm and tempo. In D. Deutsch (Ed.), The psychology of music (pp. 149-180). New York: Academic Press.

GARNER, W. R. (1974). The processing of information and structure. Potomac, MD: Erlbaum.

HANDEL, S. (1974). Perceiving melodic and rhythmic auditory patterns. Journal of Experimental Psychology, 103, 922-933.

HANDEL, S. (1989). Listening: An introduction to the perception of auditory events. Cambridge, MA: MIT Press.

HANDEL, S., LAWSON, G. R. (1983). The contextual nature of thythmic interpretation. Perception \& Psychophysics, 34, 103-120.

Hirsh, I. J., Monahan, C. B., Grant, K. W., Singh, P. G. (1990) Studies in auditory timing: 1 . Simple patterns. Perception \& Psychophysics, 47, 215-226.

LERDAHL, F., JACKENDOFF, R. (1983). A generative theory of tonal music. Cambridge, MA: MIT Press.

LongueT-Higgins, H. C., LEe, C. S. (1982). The perception of musical rhythms. Perception, 11, 115-128.

Longuet-Higgins, H. C., \& LeE, C. S. (1984). The rhythmic interpretation of monophonic music. Music Perception, 1, 424-441.

Monahan, C. B., HirSh, I. J. (1990). Studies in auditory timing: 2. Rhythm patterns. Perception \& Psychophysics, 47, 227-242.

Palmer, C., Krumhansl, C. L. (1990). Mental representations for musical meter. Journal of Experimental Psychology: Human Perception \& Performance, 16, 728-741.

Povel, D. -J. (1981). Internal representation of simple temporal patterns. Journal of Experimental Psychology: Human Perception \& Performance, 7, 3-18.

Povel, D. -J., \& Essens, P. (1985). Perception of temporal patterns. Music Perception, 3, 411-440.

Povel, D. J., \& OKKerman, H. (1981). Accents in equitone sequences. Perception \& Psychophysics, 30, 565-572.

SANDER, F. (1930). Structure, totality of experience, and gestalt. In C. Murchison (Ed.), Psychologies of 1930 (pp. 188-204). Worchester, MA: Clark University Press.

Smith, G. (1957). Visual perception: An event over time. Psychological Review, 64, 306-313.

TEN HoOPEN, G. (1992, February). Temporal processing of fast auditory patterns. Paper presented at the 2 nd International Conference on Music Perception and Cognition, Los Angeles, CA.

(Manuscript received December 12, 1991; revision accepted for publication May 3, 1992.) 